\title{
Crystallographic Analysis of Rock Grain Orientation at Meso- and Microscale Levels
}

\author{
A. S. Kulkov ${ }^{1,2, a)}$, A. I. Chernishov ${ }^{1, b)}$, D. V. Lychagin ${ }^{1,3, c)}$, \\ P. A. Tishin ${ }^{1, d)}$, and S. N. Kulkov ${ }^{1,2, e)}$ \\ ${ }^{1}$ National Research Tomsk State University, Tomsk, 634050, Russia \\ ${ }^{2}$ Institute of Strength Physics and Materials Science SB RAS, Tomsk, 634055, Russia \\ ${ }^{3}$ National Research Tomsk Polytechnic University, Tomsk, 634050, Russia \\ a) Corresponding author: 727@sibmail.com \\ b) aich@ggf.tsu.ru \\ c)dvl-tomsk@mail.ru \\ d) tishin_pa@mail.ru \\ e) kulkov@ms.tsc.ru
}

\begin{abstract}
This paper studies the results of electron backscatter diffraction analysis of naturally deformed polycrystalline olivine. It also defines the dependence of lattice-preferred orientations of grains on their microstructural position and size. The authors detect the basic mechanisms, consequence and thermal dynamic modes of deformation. They also show that the development of a polycrystalline structure is determined by the following consecutive activation of sliding systems $(010)[100] \rightarrow\{0 \mathrm{kl}\}[100] \rightarrow(100)[010] \rightarrow\{100\}[001] \rightarrow\{110\}[001]$, when dislocation sliding and diffusion creep change under the temperature decrease from $1000^{\circ} \mathrm{C}$ to $650^{\circ} \mathrm{C}$.
\end{abstract}

Keywords: EBSD, olivine, plastic deformation, lattice-preferred orientation

\section{INTRODUCTION}

Rocks are complicated multicomponent systems formed due to the interconnected processes of crystallization, solid-state reactions, plastic deformations, and other physico-chemical processes. It promotes significant convergence of characteristics that makes it possible to unambiguously define the mechanisms of their structure development. Consequently, monogene (single-phase) aggregates are the tool of choice for defining the deformation evolution of rocks, since the role of solid-state reactions is minimal in this case. One of such aggregates is dunite, which is $90 \%$ olivine $\left((\mathrm{Mg}, \mathrm{Fe})_{2} \mathrm{SiO}_{4}\right)$ that crystallizes in an orthorhombic crystal system and belongs to the $(\mathrm{mmm})$ symmetry group. Dunites are characterized by high plasticity implemented within the temperature range from $400^{\circ} \mathrm{C}$ to $1200^{\circ} \mathrm{C}$ and pressure range from 0.2 to $2 \mathrm{GPa}[1,2]$. These ranges cover the entire vertical extent of the lithosphere, thus, deformational microstructures of dunites demonstrate the stressed condition of different crust and upper mantle levels. Therefore, there has been a great interest in studying the mechanisms of olivine plastic deformations for over 50 years [1-5]. One of the main directions for research in this area is the analysis of artificially deformed mono- and polycrystalline minerals of olivines by optic methods and electron microscopy [5, 6]. This solves a direct problem of studying the structural change of a material being under certain impact conditions. The solution to the inverse problem is based on studying the microstructure of naturally deformed olivine and directed at reconstructing the stressed condition of the lithosphere with due consideration of the temperature change [3-6]. Until recently, solving the inverse problem has been limited to using optic methods only, which are based on the analysis of morphology and special characteristics of grain orientation in transmitted light appropriate for a grain no smaller than $0.1 \mathrm{~mm}$. The invention of the electron backscatter diffraction (EBSD) method allows lowering this threshold down to 1 micrometer. It provides for more statistical data and allows obtaining new scientific knowledge on the boundary types and micro grain orientations.

International Conference on Physical Mesomechanics of Multilevel Systems 2014

AIP Conf. Proc. 1623, 323-326 (2014); doi: 10.1063/1.4898947

(C) 2014 AIP Publishing LLC 978-0-7354-1260-6/\$30.00 


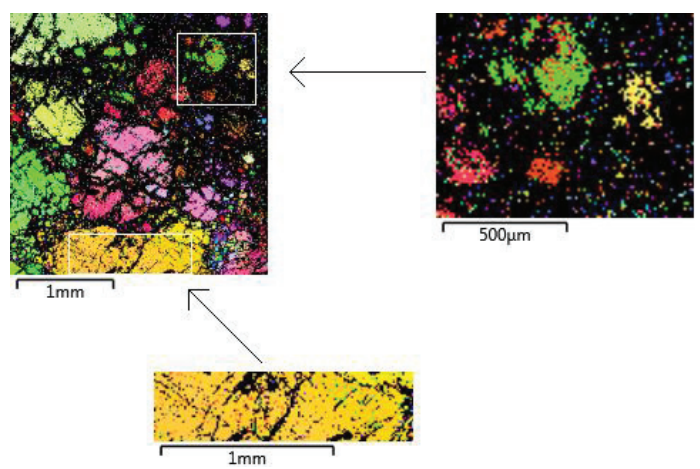

FIGURE 1. Misorientation analysis throughout the sample and on two chosen fragments

When the methods of optic microscopy are combined with EBSD, this facilitates the development of a multilevel approach to studying the morphological characteristics as well as the specific features of spatial orientation connected with them. The aim of this work is to define the deformation mechanisms connected with the hierarchy of grain and sub-grain patterns of naturally deformed olivine.

\section{MATERIALS AND METHODS}

The object of this study was a plastically deformed olivine sample. Its fabric is defined by the presence of grains of three size levels that differ from each other by almost an order of magnitude. The olivine grain structure was analyzed with an optical polarizing microscope (Leica DM 2500P). Using this equipment, we calculated the average size of the olivine grains, as well as the percentage ratio of large and small compounds that reflect the main processes of high-temperature deformations.

The crystallographic orientation of grains was studied using a scanning electron microscope (Tescan Vega II LMU) in the EBSD (Electron backscatter diffraction) mode. We analyzed the grain orientations of the material under study by plotting direct pole figures and by means of grain orientation mapping. The sample area was about $1 \mathrm{~cm}^{2}$. It contained a representative set of each grain size (particle size). The amount of processed points (where the readings are taken) varies from 1500 to 2500 . The EBSD method orientation error is no greater than $0.1^{\circ}$ and the locality is $1 \mu \mathrm{m}^{2}$.

\section{RESULTS AND DISCUSSION}

The sample structure is represented by an aggregate of three typical medium-size grains: type 1 refers to a grain size ranging from 0.7 to $2 \mathrm{~mm}$; type 2 , to a grain size from 0.1 to $0.5 \mathrm{~mm}$; and type 3 , to a grain size from 0.01 to $0.1 \mathrm{~mm}$. Moreover, we can see a trend here: the further away a grain is from the largest grain boundaries, the smaller it is in size. The biggest crystals are found to have characteristics of a subgrain structure. For this grain set, we conducted a misorientation analysis throughout the sample and on two chosen fragments with the largest grains and a fine-grain matrix (Fig. 1).

Spatial orientation for the total grain set demonstrates the marked axes maximum [100] and axis scattering belts [010] and [001] corresponding to the said maximum. Meanwhile, the whole picture becomes more complicated due to the local misorientation of crystallographic axes and formation of secondary belts (Fig. 2(a)).

Spatial grain position of a large-grain fragment is defined by the orientation of the three main individuals, which are turned around the axis away from each other [100] (Fig. 2(b)). Moreover, the largest individual does not show marked axis scattering belts [010] and [001]. This indicates that stresses appear at the initial stage of deformation and relax during the translational slip along the system (010)[100].

The second-type grains demonstrate correspondence of the main concentration positions to a large-crystal aggregate. However, the picture looks more complicated due to misorientations over the field with angular limits of $20^{\circ}$ (Fig. 2(c)). Such an orientation indicates the inheritance of the main deformation mechanisms initiated as a result of the translational slip along the $\{0 k l\}[100]$ systems with the following shear activation along the (100)[010] slip system. Orientation of the third-type grains is characterized by the arch-type scattering of axes [100] and [001] as well as feebly marked orthogonal maximum [010], which indicates the activation of slip systems $\{100\}[001]$ and $\{110\}[001]$. 


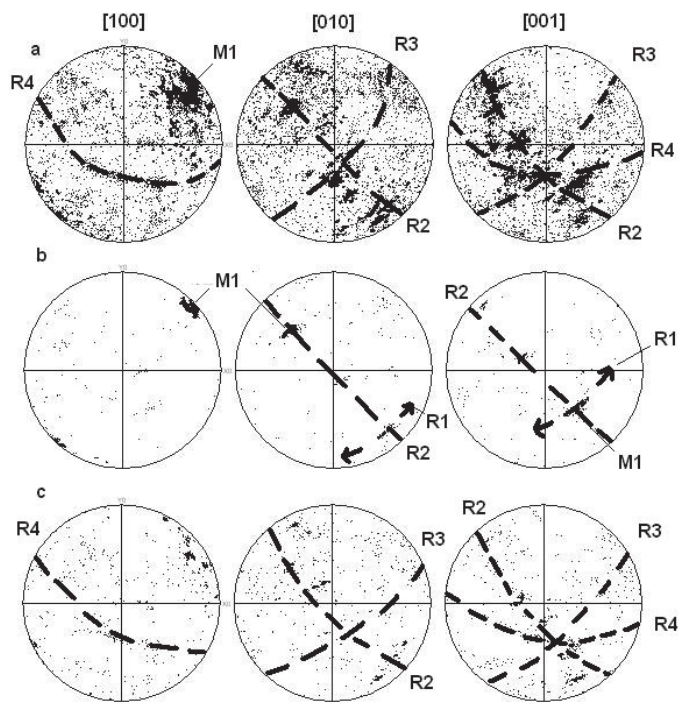

FIGURE 2. Direct pole shapes of dunite: (a) whole sample area, (b) min grain size, c) max grain size, M1 is maximum, R1-R4 ranges of orientation

Thus, it is shown that the deformation of the largest grains (type 1) is implemented by a consecutive transition of slip system (010)[100] into $\{0 \mathrm{kl}\}[100]$. Meanwhile, the $\{0 \mathrm{kl}\}[100]$-type systems prevail. Transition to slip system (010)[100] dramatically restricts the opportunities of grain reshaping under stresses. Pole figure analysis has demonstrated that the action of systems $\{0 \mathrm{kl}\}[100]$ is restricted in the second grain type (type 2), and system (100)[010] is activated. The inclusion of completely new systems $\{100\}[001]$ and $\{110\}[001]$ is typical of the third grain type (type 3 ) (transition from pinacoid to prismatic systems).

The experimental data $[1,7,8]$ allows us to assume that the consecutive activation of slip systems that was set $(010)[100] \rightarrow\{0 k l\}[100] \rightarrow(100)[010] \rightarrow\{100\}[001] \rightarrow\{110\}[001]$ reflects the progressive decrease of deformation temperatures.

It is also confirmed by the calculations of the phase equilibrium temperature in an olivine-chromium-spinel system. The authors used J. Fabries' thermometer [9] for their calculations, which showed that the temperatures of olivine formation decrease from $950{ }^{\circ} \mathrm{C}$ (for the first grain type) down to $650^{\circ} \mathrm{C}$ (for the third grain type)

Analysis of the active slip systems in different grain types, their spatial position and morphological characteristics allowed us to define and study the associated deformation mechanisms and conditions of the process. According to the work of Tsukahara et al., 1976 [5], the mechanism of olivine deformation depends on the amount of stress applied. At high stresses, dislocation slip prevails. At low stresses, dislocation creep is dominant. In all the cases, temperature plays an important role. Temperature can affect the activation of recovery, recrystallization, and diffusion processes during the deformation.

The results of structural analyses presented in the work indicate the importance of olivine recrystallization along with the process of dislocation slip inside a grain. The overview of papers on olivine deformation [10] implies that at temperatures exceeding that of initial slip along $\{0 \mathrm{kl}\}[100]$, by over $50^{\circ}$, we do not only observe the relaxation but also the formation of sub grains. Consequently, a temperature increase triggers the recrystallization processes during deformation. Chernyshov [11] observed similar processes in 2005, when he studied a group of olivines by the transmission electron microscopy method. It can be assumed that the development of recrystallization leads to forming a finer-grain fraction. In this case, low stresses and significant durations of the process create favorable conditions for diffusion transition and the activation of diffusional creep mechanism. Authors of [12] noted an important role of oxygen ion diffusion in structural transformations in olivines and mechanisms controlling a deformation process. Taking this into consideration, the authors suppose that the power law of creep is controlled by oxygen diffusion, which diffuses as separate ions but not as a compound of $\mathrm{SiO}_{4}{ }^{4-}$ complex. The above mentioned mechanism implies a sudden activation of the role of water in the olivine recrystallization process. We confirmed this by discovering the change of translational slip vectors from [100] and [010] in the first- and second-type grains to $[001]$ in the third-type grains. 
According to experimental data and calculations, such structure-forming transition can be implemented at temperatures below $850^{\circ} \mathrm{C}$ only if water participates in the reaction $[13,14]$.

Grain size is very important for analyzing creep deformation mechanisms. As it was mentioned above, dynamic recrystallization at a high temperature can cause the formation of a finer-grain fraction in olivines. Simultaneously with a grain size change, a change in a creep mechanism can occur. Each grain type will have its own or dominant creep mechanism. With a decrease in the grain size during polycrystalline deformation, the influence of grain boundary slip is expected to grow through the diminishing role of intragranular slip.

\section{CONCLUSION}

The study of naturally deformed polycrystalline olivine has shown that progressive deformation promotes progressive decrease of the grain size. At the same time, crystallites of finite deformation differ in size from initial grains by two orders of magnitude. Applying the electron backscatter diffraction (EBSD) method allowed us to define the interaction between different-size grains. We have established that subgrain structures are formed in primary grains at the initial deformation stage due to the translational slip along the (010)[100] system at the temperature of about $1000^{\circ} \mathrm{C}$. They occurred in the deep mantle conditions under very high temperatures and slow deformation, and they show the rheological mode of lower lithospheric layers. The subsequent deformations are implemented by dynamic recrystallization during a translational slip along the systems $\{0 \mathrm{kl}\}[100] \rightarrow(100)[010]$. These stages are notable for the formation of new grains with the size ranging from 0.1 to $0.5 \mathrm{~mm}$ and reflect the mechanism of dislocation creep being replaced by that of diffusion creep at temperatures ranging from $850^{\circ} \mathrm{C}$ to $1000^{\circ} \mathrm{C}$. This mode is implemented at lower parts of the earth's crust under the conditions of relatively high decreasing temperatures and faster deformation. At final stages of plastic deformations, the structure-forming role of sliding along grain boundaries (grain boundary sliding) grows due to the diminishing influence of intergranular sliding along the systems (100)[001] and $\{110\}[001]$. These processes are assumed to be caused by diffusion creep at the temperatures of $850^{\circ} \mathrm{C}$ or higher and the active participation of water fluid. These parameters correspond to the lower temperature limit of olivine resistance and indicate that its deformation mode occurred in upper crust levels.

\section{ACKNOWLEDGEMENTS}

The authors are grateful for the partial financial support of the Competitiveness Improvement Program of the Leading Research Tomsk State University and RSF agreement No. 14-17-00198. The investigations were carried out using the equipment of the Analytical Center for Geochemistry of Natural Systems at TSU.

\section{REFERENCES}

1. A. Nicolas and J. P. Poirier, Crystalline Plasticity and Solid State Flow in Metamorphic Rocks (WileyInterscience, New York, 1976).

2. V. A. Simonov, A. I. Goncharenko, A. I. Chernyshov, "Evolution of the naturally deformed dunite fabric upon melting under oriented compression", 4, 119 (1988).

3. H. Jung and S. Karato, Science 293(5534), 1460 (2001).

4. H. Jung and S. Karato, J. Struct. Geol. 23, 1337 (2001).

5. A. I. Chernyshov and A. N. Yurichev, Western Sayan. 4, 266 (2013).

6. S. N Kulkov, A. S. Kulkov, and A. I. Chernyshov, "Petrographic and X-ray chrystallographic analysis of plastically deformed dunites", S1, 83 (2010).

7. H. G. Avé Lallemant and N. L. Carter, Bull. Geol. Cos. Amer. 81, 2203 (1970).

8. N. L. Carter, Rev. Geophys. Space Phys. 14(3), 301 (1976).

9. J. Fabries, Contrib. Miner. Petrol. 69(4), 329 (1979).

10. I. Katayama, H. Jung, and S. Karato, Geology 32(12), 1045 (2004).

11. A. I. Chernyshov, Russ. Geol. Geophys. 46, 1103 (2005).

12. C. W. Passchier and R. A. J. Trouw, Microtectonics (2005).

13. S. J. Mackwell, D. L. Kohlstedt, and M. S. Paterson, J. Geophys. Res. 90, 11319 (1985).

14. H. Jung, I. Katayama, Z. Jiang, T. Hiraga, and S. Karato, Tectonophysics 421, 1 (2006). 